\title{
The Hubble Catalog of Variables (HCV) (Corrigendum)
}

A. Z. Bonanos ${ }^{1}$, M. Yang ${ }^{1}$, K. V. Sokolovsky ${ }^{1,2,3}$, P. Gavras ${ }^{4,1}$, D. Hatzidimitriou ${ }^{1,5}$, I. Bellas-Velidis ${ }^{1}$, G. Kakaletris ${ }^{6}$, D. J. Lennon ${ }^{7,8}$, A. Nota $^{9}$, R. L. White ${ }^{9}$, B. C. Whitmore ${ }^{9}$, K. A. Anastasiou ${ }^{5}$, M. Arévalo ${ }^{4}$, C. Arviset ${ }^{8}$, D. Baines ${ }^{10}$, T. Budavari ${ }^{11}$, V. Charmandaris ${ }^{12,13,1}$, C. Chatzichristodoulou ${ }^{5}$, E. Dimas ${ }^{5}$, J. Durán ${ }^{4}$, I. Georgantopoulos ${ }^{1}$, A. Karampelas ${ }^{14,1}$, N. Laskaris ${ }^{15,6}$, S. Lianou ${ }^{1}$, A. Livanis ${ }^{5}$, S. Lubow ${ }^{9}$, G. Manouras ${ }^{5}$, M. I. Moretti ${ }^{16,1}$, E. Paraskeva ${ }^{1,5}$, E. Pouliasis ${ }^{1,5}$, A. Rest ${ }^{9,11}$, J. Salgado ${ }^{10}$, P. Sonnentrucker ${ }^{9}$, Z. T. Spetsieri ${ }^{1,5}$, P. Taylor ${ }^{9}$, and K. Tsinganos ${ }^{5,1}$

\footnotetext{
${ }^{1}$ IAASARS, National Observatory of Athens, Penteli 15236, Greece e-mail: bonanos@astro.noa.gr

Department of Physics and Astronomy, Michigan State University, East, Lansing, MI 48824, USA

Sternberg Astronomical Institute, Moscow State University, Universitetskii pr. 13, 119992 Moscow, Russia

RHEA Group for ESA-ESAC, Villanueva de la Cañada, 28692 Madrid, Spain

Department of Physics, National and Kapodistrian University of Athens, Panepistimiopolis, Zografos 15784, Greece

6 Athena Research and Innovation Center, Marousi 15125, Greece

7 Instituto de Astrofísica de Canarias, 38205 La Laguna, Tenerife, Spain

8 ESA, European Space Astronomy Centre, Villanueva de la Canada, 28692 Madrid, Spain

Space Telescope Science Institute, Baltimore, MD 21218, USA

${ }^{10}$ Quasar Science Resources for ESA-ESAC, Villanueva de la Cañada, 28692 Madrid, Spain

1 The Johns Hopkins University, Baltimore, MD 21218, USA

12 Institute of Astrophysics, FORTH, Heraklion 71110, Greece

3 Department of Physics, Univ. of Crete, Heraklion 70013, Greece

14 American Community Schools of Athens, Halandri 15234, Greece

15 Greek Research and Technology Network - GRNET, Athens 11523, Greece

16 INAF-Osservatorio Astronomico di Capodimonte, Napoli 80131, Italy
}

A\&A, 630, A92 (2019), https://doi .org/10 . 1051/0004-6361/201936026

Key words. catalogs - stars: variables: general - galaxies: active - methods: statistical - methods: data analysis - errata, addenda

Errors have occurred during the production process. On page 15 , Sect. 7, third paragraph, tenth line, the sentence should read: "The number of data points in the HCV light curves ranges from five (our cut-off limit) to 120." And the citation "Spetsieri et al.
2019" should be "Spetsieri et al., in prep.", in the first paragraph of Sect. 7.4 (page 19), and in Sect. 8, second to last paragraph (page 21). 